\title{
TO THE ISSUE OF FINDING THE STOICHIOMETRIC COEFFICIENTS IN THE CHEMICAL REACTIONS
}

\author{
Jekaterina Aleksejeva \\ Liepaja University, Latvia \\ Riga Secondary School 34, Latvia \\ Sharif Guseynov \\ Liepaja University, Latvia
}

\begin{abstract}
In the present paper, on the basis of the theory of inverse and ill-posed problems, an algorithm is proposed that allows to unambiguously determine the stoichiometric coefficients in the equations of chemical reactions of any type, including redox reactions and acid-base reactions, and, regardless of whether the constructed system of linear algebraic equations for the desired stoichiometric coefficients is underdetermined (i.e. there are fewer equations than unknowns) or overdetermined (i.e. there are more equations than unknowns). The proposed algorithm is a regularized algorithm (according to Tikhonov), which ensures that, in a computer implementation, possible computational errors will not make the comprised system of linear algebraic equations to be incapable of solving.
\end{abstract}

Keywords: chemical reaction equation; inverse problem; Tikhonov's regularization method; stoichiometric coefficients; system of linear algebraic equations.

\section{Introduction}

A chemical reaction is a process of chemical transformation of some substances (starting substances called reagents) with a specific composition and unique physical and chemical properties into other substances (final substances called products) with a different composition and other properties (for instance, see Sommer, 1975; Pimentel et al., 1963). For ordering and systematization of heterogeneous chemical reactions, there are several classification methods that allow them to be divided into types: by the number and composition of reagents and products; by thermal effect - endothermic (heat is absorbed) and exothermic (heat is released) reactions; by the direction of the reaction - irreversible and reversible reactions; by the presence of a catalyst - non-catalytic and catalytic reactions; by changes in oxidation states - redox reactions and reactions that are not redox. If we restrict ourselves to considering only the first of the above five main methods of classification, then the following four types of chemical reactions are distinguished: composition reaction; decomposition reaction; exchange reaction; substitution reaction. It should be emphasized that the above 
four types do not cover the entire variety of chemical reactions: for example, many important chemical reactions from the so-called redox- and acid-base reactions cannot be attributed to any of the above four types. Finally, we note that there are many chemical reactions that can simultaneously be a redox reaction and a composition reaction, or a decomposition reaction, or a substitution reaction, but no chemical reaction can simultaneously be an exchange reaction and a redox reaction - all exchange reactions are included to the set of reactions proceeding without changing the oxidation states of the elements that form reagents and reaction products. To record chemical reactions, it is convenient to use abbreviated formulas of substances (both reagents and products). If the substances participating in a chemical reaction consist of molecules, then such formulas of substances are called not just abbreviated formulas, but molecular formulas. For example, $\mathrm{H}_{2}$ is the molecular formula of hydrogen, $\mathrm{Cl}_{2}$ - of chlorine, $\mathrm{CO}_{2}-$ of carbon dioxide, $\mathrm{N}_{2}$ - of nitrogen, $\mathrm{H}_{2} \mathrm{O}$ - of water, $\mathrm{N}_{2} \mathrm{H}_{4}$ - of hydrazine, etc. The number below to the right of the symbol of a chemical element is called an index, it shows how many atoms of a given element are contained in a molecule (index 1 is not written).

A chemical reaction equation is a conditional notation of a chemical reaction using abbreviated formulas for substances, numerical coefficients called stoichiometric coefficients, and some mathematical symbols, in particular, the arithmetic operation of addition. The composition of the equation of a chemical reaction is based on the laws of stoichiometry, first of all, on the law of conservation of the mass of substances in chemical reactions. As a rule, the left side of the chemical reaction equation consists of the formulas of the reagents, and the right side of the formulas of the reaction products. Thus, the equation of a chemical reaction provides qualitative and quantitative information about the chemical reaction, reagents and reaction products. In general, the chemical reaction equation has the form

$$
\sum_{j=1}^{N} s_{j} R_{j} \rightarrow \sum_{j=1}^{M} s_{n+j} P_{j}
$$

in which by $N \in \mathbb{N}$ and $M \in \mathbb{N}$ we denote natural numbers, representing respectively the total number of reactants $R_{j}, j=\overline{1, N}$ involved in the reaction and the total number of products $P_{j}, j=\overline{1, M}$ formed in the reaction; $s_{j}, j=\overline{1, N+M}$ denote the so-called stoichiometric coefficients, which are natural numbers, the essence of which is to ensure the equality of the number of atoms of each chemical element in the left and right sides of the chemical equation, because according to the law of conservation of mass, the number of identical atoms in different parts 
of the chemical equation must coincide (if the stoichiometric coefficient equals 1 , then it is not written).

One of the tasks in a chemistry course is to determine the stoichiometric coefficients $s_{j}, j=\overline{1, N+M}$ in equations of the form (1) of chemical reactions of all types. There are various approaches for determining stoichiometric coefficients (for instance, see Abkin, 1971, that is a guide for teachers, where these approaches are expounded sufficiently). As far as the authors of this paper are aware, the so-called algebraic method of drawing up the equations of chemical reactions was first described in detail in the posthumous work (Krapivin, 1929) of Krapivin Sergei Gavrilovich (03.16.1868 - 09.05.1927), the professor of the Department of Chemistry of the Physics and Mathematics Faculty of Lomonosov Moscow State University, who was a follower (main area of scientific interests: organic and physical chemistry, chemical kinetics) of the world famous RussianSoviet scientist, organic chemist, member of the Academy of Sciences Nikolai Dmitrievich Zelinsky (25.01.1861 - 31.07.1953). It should be noted that Professor S.G.Krapivin was sceptical about the catholicity of this method for determining the stoichiometric coefficients in the equations of chemical reactions, especially in relation to redox reactions and acid-base reactions. Roughly within the next 30 years, this method of drawing up the equations of chemical reactions was rarely discussed in the methodological literature, and mainly in a negative way. However, starting from the 60 s of the XX century, some authors - both chemistry scientists and school teachers have made active attempts to revive this method (for instance, see Berg et al., 1959; Khrustalev, 1968; Polyakova, 1969; Kuznetsova \& Adrienko, 1976; Blakley, 1982; Adamishvili \& Gambashidze, 1984). Along with these attempts, there have appeared serious works, including those of a polemical nature (for instance, see Abkin, 1971), in which numerous examples were given to illustrate the significant limitations of this method (counterexamples were constructed in which the algebraic method gives incorrect results even when applied to substitution reactions). It was summed up that if in the considered equation of a chemical reaction the number of desired stoichiometric coefficients (i.e. the number of molecules - the number of substances) is 4 more than the number of equations (i.e. the number of participating chemical elements - reagents and products), then the algebraic method of drawing up the balance becomes rather time consuming or even crippled. And, therefore, a natural question arises: why are formal complex calculations needed being divorced from the chemical contents?

The aim of the present paper is as follows: on the basis of the theory of inverse and ill-posed problems (Tikhonov \& Arsenin, 1977; Andreyev \& Guseynov, 2013), to propose an algorithm that makes it possible to unambiguously determine the stoichiometric coefficients in the equations of 
chemical reactions of any type, including redox reactions and acid-base reactions, and, regardless of whether the constructed system of algebraic equations for the desired stoichiometric coefficients is underdetermined (i.e. there are fewer equations than unknowns) or overdetermined (i.e. there are more equations than unknowns). Here, we would like to emphasize that, in contrast to the algorithms from relatively recent works (Michałowska-Kaczmarczyk et al., 2015; Marinichev et al., 2014; Turchen, 2012; Sen et al., 2006; Rash \& Zurbach, 2004), the proposed algorithm is a regularized algorithm (according to Tikhonov), which ensures that, in a computer implementation, possible computational errors will not make the comprised system of algebraic equations to be incapable in solution.

Remark 1. To conclude this section, the authors would like to emphasize that this work is intended primarily for teachers teaching a traditional chemistry course, and for secondary school students studying such a course (at least within framework of the topic "Chemical Reaction Equations"). Therefore, authors of this work tried to present its scientific content in such a way that it was quite accessible for students to understand within framework of the knowledge acquired in a typical secondary school. In view of this, some complex mathematical calculations, which, as a rule, are studied within framework of the general course of higher mathematics (namely, linear algebra) and a special section of higher mathematics (namely, the theory of inverse and ill-posed problems), we will adapt and/or simplify (in general, without significant damage to mathematical completeness and rigor) to the level of algorithms (for manual or computer implementation), i.e. to the level of a finite set of precisely defined rules describing the order of actions of the performer to solve the problem. However, it should be noted that it is quite possible that not in all secondary schools in Latvia (or other countries) senior students are familiar with the elements of higher mathematics, in particular, with systems of linear algebraic equations, methods for solving them, and problems that arise. Nevertheless, authors of the present paper surely know that in the 1st Riga State Gymnasium (Riga, Latvia), within framework of the course "Algebra", systems of algebraic equations are studied, in addition, a compulsory specialized course "Mathematical Analysis" is taught for students in grades 11-12; in Azerbaijan (it takes place an 11-year educational system) for students in grades 9-11 of secondary schools within framework of the subject "Mathematics", elements of higher mathematics are studied at a fairly complete level: linear algebra, analytical geometry, complex numbers, theory of limits, differential and integral calculus, etc. A similar situation takes place in Russian general educational schools (there is also an 11-year educational system). End of Remark (EOR). 


\section{System of Linear Algebraic Equations: Normal-, Underdetermined-, and Overdetermined Systems}

System of $m$ independent linear algebraic equations (for instance, see Il'in \& Poznyak, 1999)

$$
\left\{\begin{array}{l}
a_{11} \cdot z_{1}+a_{12} \cdot z_{2}+\ldots+a_{1 n} \cdot z_{n}=u_{1}, \\
a_{21} \cdot z_{1}+a_{22} \cdot z_{2}+\ldots+a_{2 n} \cdot z_{n}=u_{1}, \\
\ldots \ldots \ldots \ldots \ldots \ldots \ldots \ldots \ldots \ldots \ldots \ldots \ldots \\
a_{m 1} \cdot z_{1}+a_{m 2} \cdot z_{2}+\ldots+a_{m n} \cdot z_{n}=u_{m},
\end{array}\right.
$$

where $a_{i j}, i=\overline{1, m}, j=\overline{1, m}$, called coefficients of the system (2), and $u_{i}, i=\overline{1, m}$, called absolute terms of the system (2), are known, while $z_{j}, j=\overline{1, n}$ are $n$ unknown numbers to be determined, is called normal, underdetermined or overdetermined system, if $m=n, m<n$ or $m>n$, respectively.

If we introduce the matrix $A=\left\{a_{i j}\right\}_{i=1, m}^{j=\overline{1, n}}$ of size $m \times n$, which consists of the coefficients of the system (2); column vector $U=\left\{u_{i}\right\}_{i=1, m}$ of size $m \times 1$, which consists of absolute terms of the system (2); vector $Z=\left\{z_{j}\right\}_{j=1, n}$ of size $n \times 1$, which consists of the unknown coefficients of the system (2), then system (2) can be written in a more compact form:

$$
A Z=U .
$$

If system (3) is a normal system (the number of independent equations of system (2) coincides with the number of unknown variables, i.e. $m=n$ and, therefore, matrix $A$ of the system (3) is a square and nondegenerate matrix: $\operatorname{det}(A) \neq 0$ ), then any direct analytical method can be applied to system (3) in order to find its solution, for example (for instance, see Il'in \& Poznyak, 1999; Anton \& Rorres, 2014), Gaussian elimination method, or Cramer's rule, or inverse matrix solution (i.e. $Z=A^{-1} U$, where $A^{-1}$ is the inverse of the matrix $A, A^{-1} A=I$, $I$ is the identity matrix). Since all these direct methods can be easily studied and manually implemented by high school students, we will not describe them in this section (see the above-listed two textbooks, whose language is easy to understand even to high school students). However, here we just note that the matrix $A$ of the normal system (3) can appear to be an ill-conditioned matrix, i.e. condition 
Aleksejeva \& Guseynov, 2021. To the Issue of Finding the Stoichiometric Coefficients in the Chemical Reactions

number of matrix $A$ can be quite big: $\mu(A)=\frac{\lambda_{\max }}{\lambda_{\min }} \gg 1$, where $\lambda_{\max }$ and $\lambda_{\min }$ are maximum and minimum eigenvalues of the matrix $A$. In this case, application of direct analytical or numerical methods to the system (3) can lead to incorrect results because of two reasons: first, due to the instability of the system (3) - when solving ill-conditioned system (3) by direct methods (including the well-known the Moore-Penrose pseudoinversion method, for instance, see Gantmacher, 1967) any small errors in the initial data $A$ and/or $U$ (for example, computational errors) can lead to a situation where the found solution differs to arbitrary extent from the exact solution; secondly, if calculations are performed with finite accuracy, then in some cases it is not possible to establish whether system (3) is a degenerate, or ill-conditioned, or even inconsistent system, in other words, ill-conditioned, degenerate and inconsistent systems can be indistinguishable within the specified accuracy.

If $m \neq n$, i.e. if system (3) is underdetermined $(m<n)$ or overdetermined $(m>n)$, then without using the fundamental concept of Tikhonov regularization, no numerical methods (there are obviously no direct methods), including neither the Moore-Penrose pseudoinversion method, nor the least squares method, can provide a guaranteed stable normal solution (in the sense of the solution with the minimum norm) of system (3). Below, immediately after Remark 3, we propose an algorithm for finding a stable normal pseudosolution of system (3) in the case $m \neq n$. The proposed algorithm was first developed and justified in (Andreyev \& Guseynov, 2013) (see also the following articles, in which this algorithm was successfully applied to various economic and technical problems: Guseynov et al., 2017; Guseynov et al., 2015). The proposed algorithm is based on the fundamental ideas of Tikhonov's regularization method for solving ill-posed problems (for instance, see Tikhonov \& Arsenin, 1977).

Remark 2. Unlike direct analytical or numerical methods for solving system (3) in the case when it is a normal system and has a relatively small size (in fact, when finding stoichiometric coefficients in the equations of chemical reactions, we are dealing, as will be seen from the next section of this work, systems of linear algebraic equations of relatively small size: $m \sim 2 \div 10, n \sim 2 \div 10$ ), the regularizing algorithm proposed below requires a computer implementation: for this purpose, it seems to us, the most convenient is Mathcad software (the authors of this work implemented the proposed algorithm in Mathcad, version 14.0.0.163), which has an intuitive and easy-to-use user interface, and in which almost all mathematical symbols and operators have images familiar to all of us, which greatly facilitates the implementation (solution and analysis) of engineering and scientific calculations of various levels of complexity EOR. 
Remark 3. For the convenience of the readers, Fig. 1 shows a listing of the program in Mathcad, version 14.0.0.163, which implements the Gaussian elimination method for solving normal systems of the form (3) EOR.

The proposed algorithm for finding a stable normal pseudosolution of the joint system (3) in the case $m \neq n$ (in fact, the proposed algorithm is also applicable to the case $m=n$ ) consists of the following steps:

Step 1. In the initial data of system (3) we introduce small random or deterministic errors $\Delta=\{0 \leq h \ll 1 ; 0<\delta \ll 1\}$ (Mathcad 14 software has special built-in functions for generating random errors with various probability distributions: for instance, see Maxfield, 2009) that the resulting perturbed matrix $A^{h}$ and vector $U^{\delta}$ must satisfy the conditions $\left\|A^{h}-A\right\| \leq h,\left\|U^{\delta}-U\right\| \leq \delta$, where

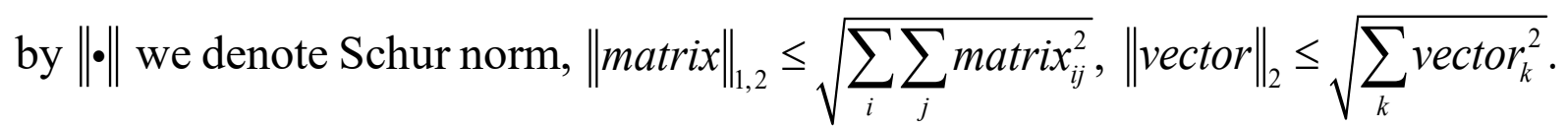

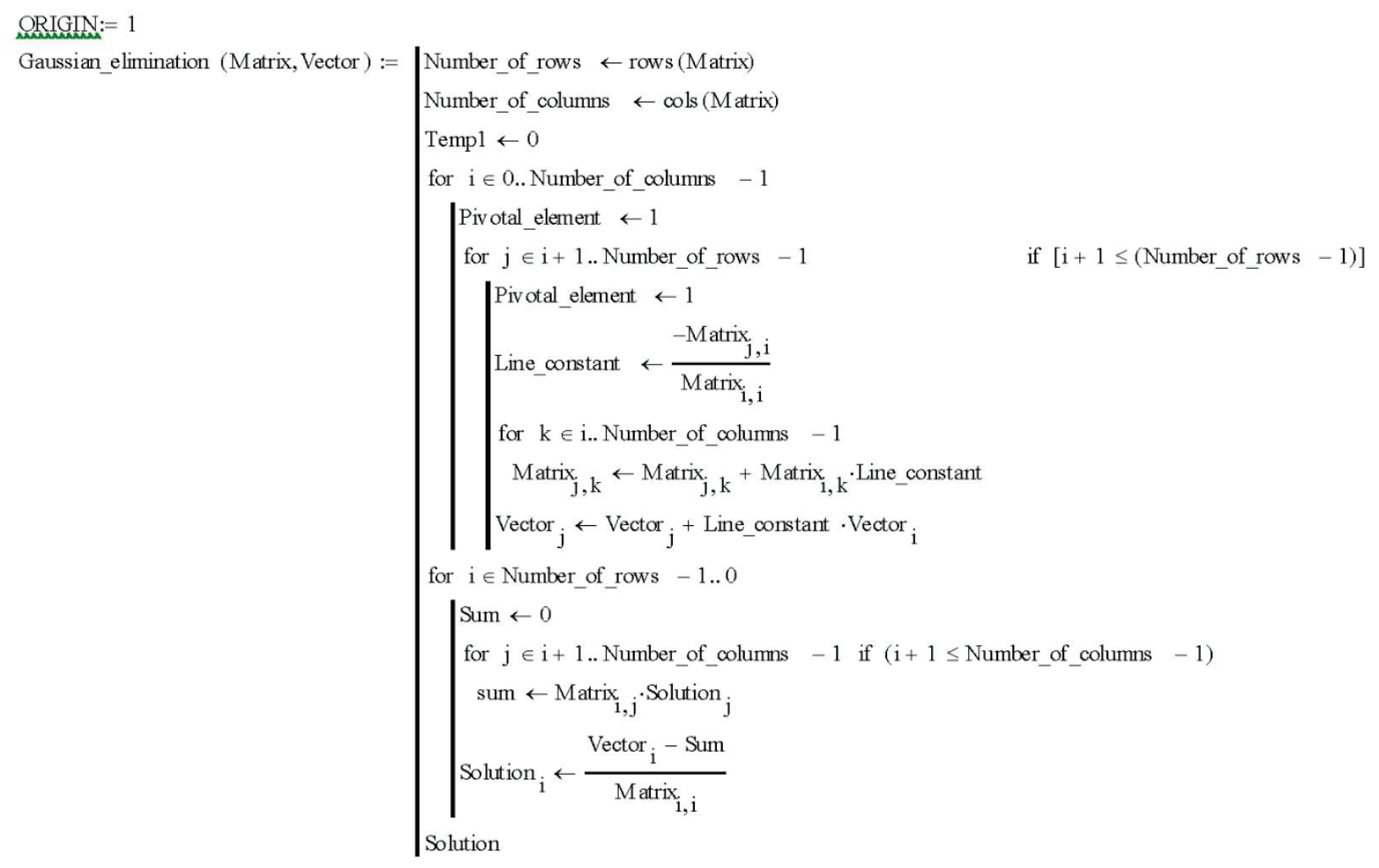

Figure 1 Listing of the Gaussian Elimination Method Implementation for Solving Normal Systems of the Kind (3)

Step 2. Instead of the original system (3), the following system is solved with respect to the unknown column vector $Z^{\alpha}$ :

$$
\left(\left(A^{h}\right)^{T} A^{h}+\alpha \cdot I\right) Z^{\alpha}=\left(A^{h}\right)^{T} U^{\delta}
$$


where $I$ is the identity matrix sized as $A^{h} ; \alpha=\alpha(\Delta)>0$ is the regularization parameter, which is defined as the solution of the following equation:

$$
\left\|A^{h} Z^{\alpha}-U^{\delta}\right\|^{2}=\frac{\left(\delta+h \cdot\left\|Z^{\alpha}\right\|\right)^{2}}{\Phi\left(m_{\varepsilon}\right)}
$$

where

$$
\left.\begin{array}{r}
\Phi\left(m_{\varepsilon}\right)=6 \cdot 10^{-6}+\left(11-m_{\varepsilon}\right) \cdot\left[0.110999+\left(1-m_{\varepsilon}\right)\right. \\
\times\left[-0.00179988+\left(6-m_{\varepsilon}\right) \cdot\left[-0.00521256+\left(9-m_{\varepsilon}\right)\right.\right. \\
\times\left[-0.718663+\left(3-m_{\varepsilon}\right) \cdot\left[-0.102426+\left(10-m_{\varepsilon}\right)\right.\right. \\
\times\left[-0.841212+\left(2-m_{\varepsilon}\right) \cdot\left[-0.163198+\left(7-m_{\varepsilon}\right)\right.\right. \\
\left.\left.\left.\left.\left.\left.\left.\times\left[-0.071326+\left(4-m_{\varepsilon}\right) \cdot\left[-0.0112364-0.00310896 \cdot\left(8-m_{\varepsilon}\right)\right]\right]\right]\right]\right]\right]\right]\right]\right], \\
m_{\varepsilon}=-\log _{10}\left(\frac{\delta}{\sqrt{m}}\right) .
\end{array}\right\}
$$

In Fig. 2 shows a listing of the program in Mathcad, version 14.0.0.163, which implements the above algorithm for finding a stable normal pseudosolution of the joint system (3) in the case $m \neq n$ (as mentioned above, the algorithm is also applicable for the case $m=n$ ).

Exercise 1. Consider a simple normal system $A Z=U$, where $A=\left(\begin{array}{cc}2 & -3 \\ -1.33 & 2\end{array}\right), U=\left(\begin{array}{c}3 \\ -1.99\end{array}\right)$. It can be verified directly that vector $Z=\left(\begin{array}{l}3 \\ 1\end{array}\right)$ is the exact solution to this system. Note that the matrix $A$ is an ill-conditioned matrix: $\mu(A) \approx 1877$. We perturb only the right side of this system by $\delta=\left(\begin{array}{l}10^{-2} \\ 10^{-2}\end{array}\right)$ : we get the system $A Z^{\delta}=U^{\delta}$, where $U^{\delta}=\left(\begin{array}{c}3.01 \\ -1.98\end{array}\right)$. Therefore, the absolute and relative errors of the introduced deviations are $\left\|U-U^{\delta}\right\| \approx 0.014$ and $\frac{\left\|U-U^{\delta}\right\|}{\left\|U^{\delta}\right\|} \approx 3.93 \cdot 10^{-3}$, respectively, i.e. the original and perturbed systems are very close. It would seem that their solutions should also be close. However, the standard packages of applied programs Mathcad 14, MATLAB R2007, MAPLE 
16 and MATEMATICA 8 (only these softwares were available to the authors of this work) find that the solution of the perturbed system is the vector $Z^{\delta} \approx\left(\begin{array}{c}8 \\ 4.33\end{array}\right)$.

ORIGIN: $=1$

\section{Schur norm}

$\operatorname{norm}($ Matrix $):=\mid \begin{aligned} & \mathrm{a} \leftarrow \operatorname{rows}(\text { Matrix }) \\ & \mathrm{b} \leftarrow \operatorname{cols}(\text { Matrix }) \\ & \text { Temp } \leftarrow \sqrt{\sum_{\mathrm{i}=1}^{\mathrm{a}} \sum_{\mathrm{j}=1}^{\mathrm{b}}\left(\text { Matrix }_{1, j}\right)^{2}}\end{aligned}$

Perturbation of the right-hand side

$\mathrm{U}_{-} \delta:=\mid \begin{aligned} & \text { for } \mathrm{i} \in 1 . . \operatorname{rows}(\mathrm{U}) \\ & \mathrm{U}_{-} \delta_{\mathrm{i}} \leftarrow \mathrm{U}_{\mathrm{i}}+\operatorname{mnd}(0.2) \\ & \mathrm{U}_{-} \delta\end{aligned}$

Perturbation of the main matrix

Absolute and Relative Errors

A_h : $=\mid \begin{aligned} & \text { for } i \in 1 . . \operatorname{rows}(A) \\ & \text { for } j \in 1 . . \operatorname{cols}(A) \\ & \quad A_{-} h_{i, j} \leftarrow A_{i, j}+\text { mnd }(0.2) \\ & \text { A_h }\end{aligned}$

Abs_error_Matrix $:=\operatorname{norm}\left(\mathrm{A}-\mathrm{A} \_\mathrm{h}\right) \quad$ Rel_error_Matrix $:=\frac{\text { Abs_error_Matrix }}{\operatorname{norm}\left(\mathrm{A} \_\mathrm{h}\right)}$

Abs_error_RHS $:=\operatorname{norm}\left(U-U_{-} \delta\right) \quad$ Rel_error_RHS $:=\frac{\text { Abs_error_RHS }}{\operatorname{norm}\left(U_{-} \delta\right)}$

Guaranteeing of inequalities $\| u_{-}$exact $-u_{-} \delta \| \mid<=\delta$ and $\| A_{-}$exact $-A_{-} h \|<=h$

$\delta$ max $:=$ Abs_error_RHS $\cdot\left(1+10^{-10}\right)$ h_max $:=$ Abs_error_Matrix $\cdot\left(1+10^{-10}\right)$

\section{Regularization algorithm}

Given

A_h $h(\alpha):=A_{-} h^{\mathrm{T}} \cdot \mathrm{A} \_\mathrm{h}+\alpha \cdot$ identity $\left(\operatorname{cols}\left(\mathrm{A} \_\mathrm{h}\right)\right)$

$z_{-} \alpha(\alpha):=\operatorname{lsolve}\left(A_{-} h \alpha(\alpha), A_{-} h^{T} \cdot U_{-} \delta\right)$

$\mathrm{m}_{-} \varepsilon:=-\log \left(\frac{\delta \_\max }{\text { rows }\left(\mathrm{U}_{-} \delta\right)}\right)$

$\Phi\left(\mathrm{m}_{-} \varepsilon\right):=\left[6 \cdot 10^{-6}+\left(11-\mathrm{m}_{-} \varepsilon\right) \cdot\left[0.110999+\left(1-\mathrm{m}_{-} \varepsilon\right) \cdot\left[-0.00179988+\left(6-\mathrm{m}_{-} \varepsilon\right) \cdot[-0.00521256+\right.\right.\right.$

$F 1(\alpha):=\left(\operatorname{norm}\left(A_{-} h \cdot z_{-} \alpha(\alpha)-U_{-} \delta\right)\right)^{2}-\frac{\left(\delta \max +h_{-} \max \cdot \operatorname{norm}\left(z_{-} \alpha(\alpha)\right)\right)^{2}}{\Phi\left(m_{-} \varepsilon\right)}$

$\alpha:=(\operatorname{root}(\mathrm{F} 1(\alpha), \alpha, 0,1))$

Figure 2 Listing of the Proposed Regularizing Algorithm for Finding a Stable Normal Pseudosolution of the System (3) in the Case $n \neq m$ 
A similar result is obtained by using the Gaussian elimination method. Consequently, the absolute and relative errors of the solution caused by introduction of a sufficiently small inaccuracy in the right-hand side (with a relative error $3.93 \cdot 10^{-3}$ ), are $\left\|Z-Z^{\delta}\right\| \approx 6.007$ and $\frac{\left\|Z-Z^{\delta}\right\|}{\left\|Z^{\delta}\right\|} \approx 0.66$, respectively. Application of the above-described regularizing algorithm (4)-(6) gives the following results (implemented in Mathcad, version 14.0.0.163): stable normal pseudosolution is a vector $Z_{\text {Reg. }}^{\alpha} \approx\left(\begin{array}{l}2.99997 \\ 0.99921\end{array}\right)$ (which is solution of the system (4)) which is achieved with the regularization parameter $\alpha \approx 1.0492659355210154 \cdot 10^{-5}$ (which is root of the equation (5)). In this case, the absolute and relative errors of the found pseudosolution are $\left\|Z-Z_{\text {Reg. }}^{\alpha}\right\| \approx 7.9 \cdot 10^{-4}$ and $\frac{\left\|Z-Z_{\text {Reg. }}^{\alpha}\right\|}{\left\|Z_{\text {Reg. }}^{\alpha}\right\|} \approx 72.51 \cdot 10^{-4}$, respectively

Exercise 2. Now consider the system $A Z=U$ with a strongly sparse matrix, where

$$
A=\left(\begin{array}{cccccccccc}
0 & 6 & 0 & 0 & 8 & 0 & 5 & 0 & 8 & 0 \\
0 & 0 & 0 & 0 & 0 & 0 & 9 & 3 & 10 & 0 \\
0 & 0 & 0 & 4 & 7 & 3 & 0 & 0 & 0 & 0 \\
0 & 0 & 0 & 0 & 0 & 5 & 6 & 0 & 9 & 0 \\
0 & 0 & 0 & 0 & 0 & 0 & 0 & 4 & 0 & 0 \\
0 & 0 & 0 & 0 & 0 & 0 & 7 & 0 & 10 & 0 \\
0 & 9 & 0 & 0 & 7 & 0 & 0 & 5 & 0 & 0 \\
0 & 0 & 6 & 0 & 0 & 0 & 0 & 0 & 0 & 0 \\
0 & 4 & 0 & 0 & 0 & 0 & 0 & 7 & 0 & 1 \\
0 & 9 & 0 & 0 & 0 & 0 & 0 & 0 & 7 & 0
\end{array}\right), U=\left(\begin{array}{c}
159 \\
177 \\
69 \\
153 \\
32 \\
139 \\
93 \\
18 \\
74 \\
81
\end{array}\right), Z=\left(\begin{array}{c}
p \\
2 \\
3 \\
4 \\
5 \\
6 \\
7 \\
8 \\
9 \\
10
\end{array}\right) .
$$

The parameter $p$ present in the exact solution indicates that the first component $Z_{1}$ the solution of this system is free (as $Z_{1}$ one can choose any number: we chose $Z_{1}=1$ ), in other words, in fact, in the considered system the number of variables $Z=\left\{z_{j}\right\}_{j=1, n}$ equals 10 (i.e. $n=10$ ), and the number of independent equations is 9 (i.e. $m=9$ ), that is, the system under consideration is a latently underdetermined system. Obviously, the determinant of the matrix $A$ 
equals $(\operatorname{det}(A)=0)$ and, therefore, no direct methods can be applied to this system, and it is impossible to solve this system on the same standard application packages listed above. We perturb the main matrix and the right side of this system by $h=0.07$ and $\delta=0.1$, respectively: then, instead of the original underdetermined system, we will have a normal system $A^{h} Z^{h, \delta}=U^{\delta}$, where $A^{h}=A+h, U^{\delta}=U+\delta$, moreover, $\operatorname{det}\left(A^{h}\right) \approx-80208$ and $\mu\left(A^{h}\right) \approx 646$. It is easy to verify that the absolute and relative errors of the deviations introduced into the main matrix and the right side of the system are $\left\|A-A^{h}\right\| \approx 0.7,\left\|U-U^{\delta}\right\| \approx 0.316$, $\frac{\left\|A-A^{h}\right\|}{\left\|A^{h}\right\|} \approx 0.02067, \quad \frac{\left\|U-U^{\delta}\right\|}{\left\|U^{\delta}\right\|} \approx 0.00089$. Application of our regularizing algorithm (4)-(6) gives the following results (implemented in Mathcad, version 14.0.0.163):

$$
\begin{gathered}
\text { - normal pseudosolution of system: } Z_{\text {Reg. }}^{\alpha} \approx\left(\begin{array}{c}
1.00144 \\
1.99999 \\
2.99996 \\
3.99999 \\
4.99999 \\
5.99999 \\
7.00003 \\
8.00001 \\
8.99997 \\
9.99947
\end{array}\right) ; \\
- \text { regularization parameter: } \alpha \approx 2.4857076795214805 \cdot 10^{-5} ; \\
- \text { absolute and relative errors: } \\
\left\|Z-Z_{\text {Reg. }}^{\alpha}\right\| \approx 1.54 \cdot 10^{-3}, \frac{\left\|Z-Z_{\text {Reg. }}^{\alpha}\right\|}{\| Z_{\text {Reg. } .}^{\alpha}} \approx 7.8 \cdot 10^{-5} .
\end{gathered}
$$

\section{Reduction of the Determining Stoichiometric Coefficients Problem to Solving a System of Linear Algebraic Equations}

As mentioned in the Introduction, if we classify chemical reactions by the number and composition of reagents $\left\{R_{j}\right\}_{j \in \mathbb{N}}$ and products $\left\{P_{j}\right\}_{j \in \mathbb{N}}$, then chemical 
reactions can refer to: the type of composition reaction, when the reagents involved in a chemical reaction combine into one product $\left(\sum_{j=1}^{N} s_{j} R_{j} \rightarrow s \cdot P\right)$; to the type of decomposition reaction, when only one reagent is involved in a chemical reaction, which decomposes to form several products $\left(s \cdot R \rightarrow \sum_{j=1}^{M} s_{j} P_{j}\right)$; to the type of exchange reaction (the "reverse exchange" is excluded), when the reactants exchange atoms with each other and even the whole constituent parts of their molecules, resulting in the formation of products, the amount of which, as a rule, coincides with the amount of reagents $\left(\sum_{j=1}^{N} s_{j} R_{j} \rightarrow \sum_{j=1}^{N} s_{n+j} P_{j}\right)$; to the type of substitution reaction, when the atoms of some reagents, which are simple substances, replace the atoms of other reagents, which are complex substances, as a result of which new products are formed - a new complex substance and a new simple substance $\left(\sum_{j=1}^{N} s_{j} R_{j} \rightarrow \sum_{j=1}^{M} s_{n+j} P_{j}\right)$.

Reagents $\left\{R_{j}\right\}_{j \in \mathbb{N}}$ and products $\left\{P_{j}\right\}_{j \in \mathbb{N}}$ in a chemical reaction (1) mathematically/symbolically are represent as $R_{j}=\left(E_{j_{1}}^{R}\right)_{R_{j_{1}}}\left(E_{j_{2}}^{R}\right)_{R_{j_{2}}} \ldots\left(E_{j_{k}}^{R}\right)_{R_{j_{k}}}$ and $P_{j}=\left(E_{j_{1}}^{P}\right)_{P_{j_{1}}}\left(E_{j_{2}}^{P}\right)_{P_{j_{2}}} \ldots\left(E_{j_{\ell}}^{P}\right)_{P_{j_{\ell}}}$, respectively, where

- $j_{k} \in \mathbb{N}, R_{j_{k}} \in \mathbb{N}$ for any $k \in \mathbb{N}$ and $j_{\ell} \in \mathbb{N}, P_{j_{\ell}} \in \mathbb{N}$ for any $\ell \in \mathbb{N}$;

- $\left(E_{j_{k}}^{R}\right)_{R_{j_{k}}}$ is $j_{k}$-th structural unit (atoms, ions, radicals, etc.) of the reagent $R_{j}, j=\overline{1, N}$ with index $R_{j_{k}}$ (as already noted in the Introduction, index shows how many atoms of a given structural unit are contained in a molecule: index 1 is not written);

- $\left(E_{j_{\ell}}^{P}\right)_{P_{j \ell}}$ is $j_{\ell}$-th structural unit of the product $P_{j}, j=\overline{1, M}$ with index $P_{j_{\ell}}$;

- $\bigcup_{j_{k}}\left(E_{j_{k}}^{R}\right)_{R_{j_{k}}}$ forms a set of unique structural units of a substance (reagents and products) in a chemical reaction, and, moreover, one can write the equality $\bigcup_{j_{k}}\left(E_{j_{k}}^{R}\right)_{R_{j_{k}}}=\bigcup_{j_{\ell}}\left(E_{j_{\ell}}^{P}\right)_{P_{j_{\ell}}}$, which means that the aggregate structural unit of reagents and the aggregate structural unit of products coincide. 
Example 1. In order to correctly understand the chemical meaning of just the introduced designations, consider a simple chemical exchange reaction in which the reagents $\left\{R_{j}\right\}_{j=1,2}$ are $\mathrm{Na}_{2} \mathrm{CO}_{3}$ and $\mathrm{HCl}$, but products $\left\{P_{j}\right\}_{j=1,3}$ are $\mathrm{NaCl}, \mathrm{H}_{2} \mathrm{O}$ and $\mathrm{CO}_{2}$, those. Consider a chemical reaction, equation (1) of which has the form:

$$
\mathrm{S}_{1} \mathrm{Na}_{2} \mathrm{CO}_{3}+\mathrm{s}_{2} \mathrm{HCl} \rightarrow \mathrm{S}_{3} \mathrm{NaCl}+\mathrm{s}_{4} \mathrm{H}_{2} \mathrm{O}+\mathrm{S}_{5} \mathrm{CO}_{2} .
$$

Then, bearing in mind that the number of unique structural units in a given chemical reaction (in reagents and products) is equal to 5 $\left(\bigcup_{j_{k}}\left(E_{j_{k}}^{R}\right)_{R_{j_{k}}}=\bigcup_{j_{\ell}}\left(E_{j_{\ell}}^{P}\right)_{P_{j_{\ell}}}=\{N a, C l, H, O, C\}\right)$, the chemical meaning of the above mathematical/symbolic representations $R_{j}=\left(E_{j_{1}}^{R}\right)_{R_{j_{1}}}\left(E_{j_{2}}^{R}\right)_{R_{j_{2}}} \ldots\left(E_{j_{k}}^{R}\right)_{R_{j_{k}}} \quad$ and $P_{j}=\left(E_{j_{1}}^{P}\right)_{P_{j_{1}}}\left(E_{j_{2}}^{P}\right)_{P_{j_{2}}} \ldots\left(E_{j_{\ell}}^{P}\right)_{P_{j_{\ell}}}$ is as follows:

$$
\begin{aligned}
& R_{1}=N a_{2} C O_{3},\left(E_{1_{1}}^{R}\right)_{R_{1_{1}}}=\underbrace{(N a)_{2}}_{E_{11}^{R}=N a ; R_{11}=2}=N a_{2},\left(E_{1_{2}}^{R}\right)_{R_{1_{1}}}=\underbrace{(C)_{1}}_{E_{1_{2}}^{R}=C ; R_{1_{2}}=1}=C,\left(E_{1_{3}}^{R}\right)_{R_{1_{3}}}=\underbrace{(O)_{3}}_{E_{13}^{R}=O ; R_{13}=3}=O_{3} ; \\
& R_{2}=H C l,\left(E_{2_{1}}^{R}\right)_{R_{21}}=\underbrace{(H)_{1}}_{E_{21}^{R}=H ; R_{21}=1}=H,\left(E_{2_{2}}^{R}\right)_{R_{22}}=\underbrace{(C l)_{1}}_{E_{22}^{R}=C l ; R_{22}=1}=C l ; \\
& P_{1}=N a C l,\left(E_{1_{1}}^{P}\right)_{P_{1_{1}}}=\underbrace{(N a)_{1}}_{E_{1_{1}}^{R}=N a ; P_{1_{1}}=1}=N a,\left(E_{1_{2}}^{P}\right)_{P_{1_{2}}}=\underbrace{(C l)_{1}}_{E_{1_{2}}^{R}=C l ; P_{1_{2}}=1}=C l \text {; } \\
& P_{2}=H_{2} O,\left(E_{2_{1}}^{P}\right)_{P_{21}}=\underbrace{(H)_{2}}_{E_{2_{1}}^{R}=H ; P_{2_{1}}=2}=H_{2},\left(E_{2_{2}}^{P}\right)_{P_{22}}=\underbrace{(O)_{1}}_{E_{2_{2}}^{R}=O ; P_{22}=1}=O \text {; } \\
& P_{3}=C O_{2},\left(E_{3_{1}}^{P}\right)_{P_{3_{1}}}=\underbrace{(C)_{1}}_{E_{3_{1}}^{R}=C ; P_{3_{1}}=1}=C,\left(E_{3_{2}}^{P}\right)_{P_{3_{2}}}=\underbrace{(O)_{2}}_{E_{3_{2}}^{R}=O ; P_{3_{2}}=2}=O_{2} ; \\
& \bigcup_{j_{k}}\left(E_{j_{k}}^{R}\right)_{R_{j_{k}}}=\bigcup\left\{E_{1_{1}}^{R}, E_{1_{2}}^{R}, E_{1_{3}}^{R}, E_{2_{1}}^{R}, E_{2_{2}}^{R}\right\}=\bigcup\{N a, C, O, H, C l\}=\{N a, C, O, H, C l\} ; \\
& \bigcup_{j_{\ell}}\left(E_{j_{\ell}}^{P}\right)_{P_{j_{\ell}}}=\bigcup\left\{E_{1_{1}}^{P}, E_{1_{2}}^{P}, E_{2_{1}}^{P}, E_{2_{2}}^{P}, E_{3_{1}}^{P}, E_{3_{2}}^{P}\right\}=\bigcup\{N a, C l, H, O, C, O\}=\{N a, C l, H, O, C\} .
\end{aligned}
$$

Obviously, in the introduced notation, equation (7) has the following notation:

$$
\begin{aligned}
& S_{1}\left(E_{1_{1}}^{R}\right)_{R_{1_{1}}}\left(E_{1_{2}}^{R}\right)_{R_{1_{2}}}\left(E_{1_{3}}^{R}\right)_{R_{1_{3}}}+s_{2}\left(E_{2_{1}}^{R}\right)_{R_{2_{1}}}\left(E_{2_{2}}^{R}\right)_{R_{22}} \rightarrow s_{3}\left(E_{1_{1}}^{P}\right)_{P_{1_{1}}}\left(E_{1_{2}}^{P}\right)_{P_{1_{2}}} \\
& +S_{4}\left(E_{2_{1}}^{P}\right)_{P_{21}}\left(E_{2_{2}}^{P}\right)_{P_{22}}+s_{5}\left(E_{3_{1}}^{P}\right)_{P_{3_{1}}}\left(E_{3_{2}}^{P}\right)_{P_{3_{2}}}
\end{aligned}
$$


Aleksejeva \& Guseynov, 2021. To the Issue of Finding the Stoichiometric Coefficients in the Chemical Reactions

This example shows that $\underbrace{E_{1_{1}}^{R}=E_{1_{1}}^{P}}_{N a}, \underbrace{E_{1_{2}}^{R}=E_{3_{1}}^{P}}_{C}, \underbrace{E_{1_{3}}^{R}=E_{2_{2}}^{P}=E_{3_{2}}^{P}}_{O}, \underbrace{E_{2_{1}}^{R}=E_{2_{1}}^{P}}_{H}$, $\underbrace{E_{2_{2}}^{R}=E_{1_{2}}^{P}}_{C l}$, i.e. out of 11 structural units (5 of them appear in reagents, and 6 in products), only 5 are unique, and this circumstance is characteristic to all chemical reactions, both the four types of reactions listed above, and redox- and acid-base reactions. In other words, for any chemical reaction in symbolic representations $R_{j}=\left(E_{j_{1}}^{R}\right)_{R_{j 1}}\left(E_{j_{2}}^{R}\right)_{R_{j_{2}}} \ldots\left(E_{j_{k}}^{R}\right)_{R_{j_{k}}} \quad$ of $\quad$ reagents $\quad\left\{R_{j}\right\}_{j=\overline{1, N}} \quad$ and $P_{j}=\left(E_{j_{1}}^{P}\right)_{P_{j 1}}\left(E_{j_{2}}^{P}\right)_{P_{j_{2}}} \ldots\left(E_{j_{\ell}}^{P}\right)_{P_{j \ell}}$ of products $\left\{P_{j}\right\}_{j=\overline{1}, M}$ the number of unique structural units will be less than the total (equal to $j_{k}+j_{\ell}$ ) structural units of $N$ reagents and $M$ products. The essence of the algebraic method for determining the stoichiometric coefficients of chemical reactions consists in drawing up a system of linear algebraic equations, in which the number of equations is equal to the number of unique structural units (which is $\operatorname{card}\left(\bigcup_{j_{k}}\left(E_{j_{k}}^{R}\right)_{R_{j_{k}}}\right)$, where $\operatorname{card}(\cdot)$ denotes the cardinality of a set) of regents and products. To construct equations of this system, it is necessary to use so-called law of material balance (for instance, see Krapivin, 1929; Abkin, 1971; Blakley, 1982; Kulibaba, 2010), which states that amount of each unique structural unit before the start of the chemical reaction is equal to the amount of the corresponding unique structural unit after the occurrence of this chemical reaction. Taking this law into account in the chemical reaction considered above with equation (8), we obtain a system of linear algebraic equations for the unknown stoichiometric coefficients of the given chemical reactions

$$
\left\{\begin{array} { l } 
{ R _ { 1 _ { 1 } } \cdot s _ { 1 } = P _ { 1 _ { 1 } } \cdot s _ { 3 } , } \\
{ R _ { 1 _ { 2 } } \cdot s _ { 1 } = P _ { 3 _ { 1 } } \cdot s _ { 5 } , } \\
{ R _ { 1 _ { 3 } } \cdot s _ { 1 } = P _ { 2 _ { 2 } } \cdot s _ { 4 } + P _ { 3 _ { 2 } } \cdot s _ { 5 } , \text { i.e. } } \\
{ R _ { 2 _ { 1 } } \cdot s _ { 2 } = P _ { 2 _ { 1 } } \cdot s _ { 4 } , } \\
{ R _ { 2 _ { 2 } } \cdot s _ { 2 } = P _ { 1 _ { 2 } } \cdot s _ { 3 } , }
\end{array} \left\{\begin{array}{l}
2 \cdot s_{1}=s_{3}, \\
s_{1}=s_{5}, \\
3 \cdot s_{1}=s_{4}+2 \cdot s_{5},(9) \\
s_{2}=2 \cdot s_{4}, \\
s_{2}=s_{3},
\end{array}\right.\right.
$$

which is easily solved manually: $s_{2}=s_{3}=2 \cdot s_{1}, s_{4}=s_{5}=s_{1}$, where $s_{1}>0$ plays the role of a free parameter, which, firstly, can always be specified as any positive number (for example, 1: then we get $s_{1}=s_{4}=s_{5}=1, s_{2}=s_{3}=2$ ), secondly, can 
always be left in equation (7) as a parameter, and then we are able to divide by it, namely, taking into account the found $s_{j}, j=\overline{1,5}$ in (7) we obtain equation $s_{1} \mathrm{Na}_{2} \mathrm{CO}_{3}+2 s_{1} \mathrm{HCl} \rightarrow 2 s_{1} \mathrm{NaCl}+s_{1} \mathrm{H}_{2} \mathrm{O}+s_{1} \mathrm{CO}_{2}$, which, after dividing both parts by $\mathrm{s}_{1}$ takes the usual "chemical" form $\mathrm{Na}_{2} \mathrm{CO}_{3}+2 \mathrm{HCl} \rightarrow 2 \mathrm{NaCl}+\mathrm{H}_{2} \mathrm{O}+\mathrm{CO}_{2}$. Application of the regularizing algorithm proposed in the previous section to system (9), which in matrix form has the form (we put $s_{1}=1$ )

$$
\underbrace{\left(\begin{array}{ccccc}
1 & 0 & 0 & 0 & 0 \\
2 & 0 & -1 & 0 & 0 \\
1 & 0 & 0 & 0 & -1 \\
3 & 0 & 0 & -1 & -2 \\
0 & 1 & 0 & -2 & 0 \\
0 & 1 & -1 & 0 & 0
\end{array}\right)}_{A_{6 \times 5}} \underbrace{\left(\begin{array}{c}
s_{1}=1 \\
s_{2} \\
s_{3} \\
s_{4} \\
s_{5}
\end{array}\right)}_{Z_{5 \times 1}}=\underbrace{\left(\begin{array}{l}
1 \\
0 \\
0 \\
0 \\
0
\end{array}\right)}_{U_{6 \times 1}},
$$

gives the following results (implemented in Mathcad, version 14.0.0.163):

- deterministically perturbed matrix and right-hand part: $A^{h}=\left\{a_{i j}+0.7\right\}_{i=1,6}^{j=\overline{1,5}}$,

$$
U^{\delta}=\left\{u_{i}+0.1\right\}_{i=1,6} ;
$$

- normal pseudosolution (solution (4)): $Z_{\text {Reg. }}^{\alpha} \approx\left(\begin{array}{c}0.99872 \\ 1.99674 \\ 1.99697 \\ 0.99837 \\ 0.99884\end{array}\right)$;

- regularization parameter (root of (5)): $\alpha \approx 1.165568962121907 \cdot 10^{-4}$;

$$
\left\|Z-Z_{\text {Reg. }}^{\alpha}\right\| \approx 5 \cdot 10^{-3}, \frac{\left\|Z-Z_{\text {Reg. }}^{\alpha}\right\|}{\left\|Z_{\text {Reg. }}^{\alpha}\right\|} \approx 1.5 \cdot 10^{-5} .
$$

Let's pay attention to the found stoichiometric coefficients (11): the question arises, how can we use the found approximate values of stoichiometric coefficients $s_{j}, j=\overline{1,5}$, if they must be natural numbers (we already know that $\left.s_{1}=s_{4}=s_{5}=1, s_{2}=s_{3}=2\right)$ ? The answer is obvious: it is necessary to round them to the nearest natural numbers, since the proposed regularizing algorithm is 
guaranteed to find a stable solution - a solution that continuously depends on the initial data, in other words, a small change in the initial data (the main matrix and the right-hand side of system (10)) is guaranteed to correspond to a small change of solution (see found absolute and relative errors).

Below are examples of more complex chemical reactions in which it is required to determine the stoichiometric coefficients by the algebraic method..

Example 2 (Blakley, 1982). Consider a chemical reaction, the equation of which has form

$$
\begin{aligned}
& \mathrm{S}_{1} \mathrm{H}_{2} \mathrm{CO}_{3}+s_{2} \mathrm{Ca}(\mathrm{CN})_{2}+s_{3} \mathrm{NaAlF}_{4}+s_{4} \mathrm{FeSO}_{4}+s_{5} \mathrm{MgSiO}_{3}+s_{6} \mathrm{KI}_{+} s_{7} \mathrm{H}_{3} \mathrm{PO}_{4} \\
& +s_{8} \mathrm{PbCrO}_{4}+s_{9} \mathrm{BrCl}+s_{10} \mathrm{CF}_{2} \mathrm{Cl}_{2}+s_{11} \mathrm{SO}_{2} \rightarrow s_{12} \mathrm{PbBr}_{2}+s_{13} \mathrm{CrCl}_{3}+s_{14} \mathrm{MgCO}_{3} \\
& +s_{15} \mathrm{KAl}(\mathrm{OH})_{4}+s_{16} \mathrm{Fe}(\mathrm{SCN})_{3}+s_{17} \mathrm{PI}_{3}+s_{18} \mathrm{Na}_{2} \mathrm{SiO}_{3}+s_{19} \mathrm{CaF}_{2}+s_{20} \mathrm{H}_{2} \mathrm{O} .
\end{aligned}
$$

The task is to determine the stoichiometric coefficients $s_{j}, j=\overline{1,20}$.

We use the aforementioned law of material balance, and, knowing that in the chemical reaction under consideration, unique structural units are $\{\mathrm{H}, \mathrm{C}, \mathrm{O}, \mathrm{Ca}, \mathrm{C}, \mathrm{N}, \mathrm{Na}, \mathrm{Al}, \mathrm{F}, \mathrm{Fe}, \mathrm{S}, \mathrm{Mg}, \mathrm{Si}, \mathrm{K}, I, P, \mathrm{~Pb}, \mathrm{Cr}, \mathrm{Br}, \mathrm{Cl}\}$, we obtain a system of linear algebraic equations (3) $A_{20 \times 20} Z_{20 \times 1}=U_{20 \times 1}$ to find the desired coefficients $Z=\left\{z_{j}=s_{j}\right\}_{j=\overline{1,20}}$ (coefficient $s_{1}>0$ we left as a free parameter), where

$$
A=\left(\begin{array}{cccccccccccccccccccc}
1 & 0 & 0 & 0 & 0 & 0 & 0 & 0 & 0 & 0 & 0 & 0 & 0 & 0 & 0 & 0 & 0 & 0 & 0 & 0 \\
2 & 0 & 0 & 0 & 0 & 0 & 3 & 0 & 0 & 0 & 0 & 0 & 0 & 0 & -4 & 0 & 0 & 0 & 0 & -2 \\
0 & 1 & 0 & 0 & 0 & 0 & 0 & 0 & 0 & 0 & 0 & 0 & 0 & 0 & 0 & 0 & 0 & 0 & -1 & 0 \\
0 & 2 & 0 & 0 & 0 & 0 & 0 & 0 & 0 & 1 & 0 & 0 & 0 & -1 & 0 & -3 & 0 & 0 & 0 & 0 \\
0 & 2 & 0 & 0 & 0 & 0 & 0 & 0 & 0 & 0 & 0 & 0 & 0 & 0 & 0 & -3 & 0 & 0 & 0 & 0 \\
0 & 0 & 1 & 0 & 0 & 0 & 0 & 0 & 0 & 0 & 0 & 0 & 0 & 0 & 0 & 0 & 0 & -2 & 0 & 0 \\
0 & 0 & 1 & 0 & 0 & 0 & 0 & 0 & 0 & 0 & 0 & 0 & 0 & 0 & -1 & 0 & 0 & 0 & 0 & 0 \\
0 & 0 & 4 & 0 & 0 & 0 & 0 & 0 & 0 & 2 & 0 & 0 & 0 & 0 & 0 & 0 & 0 & 0 & -2 & 0 \\
0 & 0 & 0 & 1 & 0 & 0 & 0 & 0 & 0 & 0 & 0 & 0 & 0 & 0 & 0 & -1 & 0 & 0 & 0 & 0 \\
0 & 0 & 0 & 1 & 0 & 0 & 0 & 0 & 0 & 0 & 1 & 0 & 0 & 0 & 0 & -3 & 0 & 0 & 0 & 0 \\
0 & 0 & 0 & 4 & 3 & 0 & 4 & 4 & 0 & 0 & 2 & 0 & 0 & -3 & -4 & 0 & 0 & -3 & 0 & -1 \\
0 & 0 & 0 & 0 & 1 & 0 & 0 & 0 & 0 & 0 & 0 & 0 & 0 & -1 & 0 & 0 & 0 & 0 & 0 & 0 \\
0 & 0 & 0 & 0 & 1 & 0 & 0 & 0 & 0 & 0 & 0 & 0 & 0 & 0 & 0 & 0 & 0 & -1 & 0 & 0 \\
0 & 0 & 0 & 0 & 0 & 1 & 0 & 0 & 0 & 0 & 0 & 0 & 0 & 0 & -1 & 0 & 0 & 0 & 0 & 0 \\
0 & 0 & 0 & 0 & 0 & 1 & 0 & 0 & 0 & 0 & 0 & 0 & 0 & 0 & 0 & 0 & -3 & 0 & 0 & 0 \\
0 & 0 & 0 & 0 & 0 & 0 & 1 & 0 & 0 & 0 & 0 & 0 & 0 & 0 & 0 & 0 & -1 & 0 & 0 & 0 \\
0 & 0 & 0 & 0 & 0 & 0 & 0 & 1 & 0 & 0 & 0 & -1 & 0 & 0 & 0 & 0 & 0 & 0 & 0 & 0 \\
0 & 0 & 0 & 0 & 0 & 0 & 0 & 1 & 0 & 0 & 0 & 0 & -1 & 0 & 0 & 0 & 0 & 0 & 0 & 0 \\
0 & 0 & 0 & 0 & 0 & 0 & 0 & 0 & 1 & 0 & 0 & -2 & 0 & 0 & 0 & 0 & 0 & 0 & 0 & 0 \\
0 & 0 & 0 & 0 & 0 & 0 & 0 & 0 & 1 & 2 & 0 & 0 & -3 & 0 & 0 & 0 & 0 & 0 & 0 & 0
\end{array}\right), U=\left(\begin{array}{c}
s_{1} \\
0 \\
0 \\
0 \\
0 \\
0 \\
0 \\
0 \\
0 \\
0 \\
0 \\
0 \\
0 \\
0 \\
0 \\
0 \\
0 \\
0 \\
0 \\
0
\end{array}\right) .
$$


Note that, despite looking sparse, matrix $A$ has a nonzero determinant, however it is an ill-conditioned matrix: $\operatorname{det}(A) \approx 1056, \mu(A) \approx 145.82$.

Solving the designed system in Mathcad software, version 14.0.0.163 using the inverse matrix solution method, $Z=A^{-1} U$ (due to the ability to carry out symbolic/algebraic calculations carried out by the operator $\rightarrow$ in Symbolic Keyword Toolbar, Mathcad allows us to operate on variables, in particular, source data that depend on free parameters: the right side $U$ of our system depends on the free parameter $s_{1}$; in addition, in Mathcad, using the "Fraction" format in the "Number Format" tab of the "Result Format" dialog box, one can present the results of numerical and symbolic calculations in fractional format), we got that $s_{2}=\frac{15}{88} \cdot s_{1}, s_{3}=\frac{3}{44} \cdot s_{1}, s_{4}=\frac{5}{44} \cdot s_{1}, s_{5}=\frac{3}{88} \cdot s_{1}, s_{6}=\frac{3}{44} \cdot s_{1}, s_{7}=\frac{1}{44} \cdot s_{1}, s_{8}=\frac{3}{44} \cdot s_{1}$, $s_{9}=\frac{3}{22} \cdot s_{1}, \quad s_{10}=\frac{3}{88} \cdot s_{1}, \quad s_{11}=\frac{5}{22} \cdot s_{1}, \quad s_{12}=\frac{3}{44} \cdot s_{1}, \quad s_{13}=\frac{3}{44} \cdot s_{1}, \quad s_{14}=\frac{3}{88} \cdot s_{1}$, $s_{15}=\frac{3}{44} \cdot s_{1}, s_{16}=\frac{5}{44} \cdot s_{1}, s_{17}=\frac{1}{44} \cdot s_{1}, s_{18}=\frac{3}{88} \cdot s_{1}, s_{19}=\frac{15}{88} \cdot s_{1}, s_{20}=\frac{79}{88} \cdot s_{1}$. Since the least common multiple for the denominators $s_{j}, j=\overline{2,20}$ is 88 , then, obviously we should take the value of the free parameter $s_{1}$ equal to this number: $s_{1}=88$. Given the already fully determined stoichiometric coefficients $s_{j}, j=\overline{1,20}$ in the equation (12), we have

$$
\begin{aligned}
& 88 \mathrm{H}_{2} \mathrm{CO}_{3}+15 \mathrm{Ca}(\mathrm{CN})_{2}+6 \mathrm{NaAlF}_{4}+10 \mathrm{FeSO}_{4}+3 \mathrm{MgSiO}_{3}+6 \mathrm{KI}+2 \mathrm{H}_{3} \mathrm{PO}_{4} \\
& +6 \mathrm{PbCrO}_{4}+12 \mathrm{BrCl}+3 \mathrm{CF}_{2} \mathrm{Cl}_{2}+20 \mathrm{SO}_{2} \rightarrow 6 \mathrm{PbBr}_{2}+6 \mathrm{CrCl}_{3}+3 \mathrm{MgCO}_{3} \\
& +6 \mathrm{KAl}(\mathrm{OH})_{4}+10 \mathrm{Fe}(\mathrm{SCN})_{3}+2 \mathrm{PI}_{3}+3 \mathrm{Na}_{2} \mathrm{SiO}_{3}+15 \mathrm{CaF}_{2}+79 \mathrm{H}_{2} \mathrm{O} .
\end{aligned}
$$

Application of the regularizing algorithm proposed in the previous section to this problem (we "traditionally" put $s_{1}=1$, not $s_{1}=88$ ) gives the following results (implemented in Mathcad, version 14.0.0.163):

- randomly perturbed matrix and right-hand side:

$A^{h}=\left\{a_{i j}+\operatorname{rnd}(0.2)\right\}_{i, j=1,20}, U^{\delta}=\left\{u_{i}+\operatorname{rnd}(0.2)\right\}_{i=1,20}$,

here the built-in function $\operatorname{rnd}(x)$ generates a uniformly distributed random number between 0 and $\mathrm{x}$;

- normal pseudosolution (i.e., solution of the system (4), see Fig. 2): 
Aleksejeva \& Guseynov, 2021. To the Issue of Finding the Stoichiometric Coefficients in the Chemical Reactions

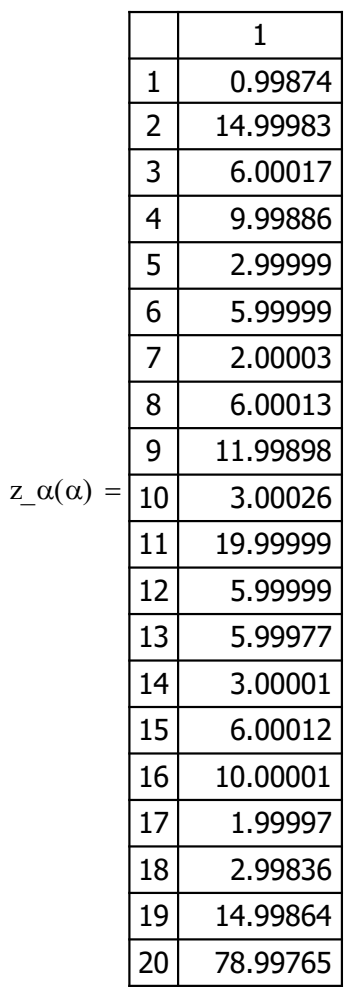

- regularization parameter (root of (5)): $\alpha \approx 1.165568962121907 \cdot 10^{-4}$;

- absolute and relative errors:

$$
\left\|Z-Z_{\text {Reg. }}^{\alpha}\right\| \approx 3.77 \cdot 10^{-3}, \frac{\left\|Z-Z_{\text {Reg. }}^{\alpha}\right\|}{\left\|Z_{\text {Reg. }}^{\alpha}\right\|} \approx 4.3 \cdot 10^{-5} .
$$

As mentioned above, the coordinates of the obtained normal pseudosolution vector must be rounded to the nearest natural numbers.

Example 3 (Kulibaba, 2010). Consider chemical reactions that are described by the following equations:

$$
\begin{gathered}
s_{1} \mathrm{KNO}_{3}+s_{2} \mathrm{~S} \rightarrow \mathrm{s}_{3} \mathrm{~K}_{2} \mathrm{SO}_{4}+s_{4} \mathrm{KSO}_{3}+s_{5} \mathrm{KNO}_{2}+s_{6} \mathrm{SO}_{3}+s_{7} \mathrm{NO}+s_{8} \mathrm{~N}_{2}+s_{9} \mathrm{O}_{2}+s_{10} \mathrm{SO}_{2} \\
s_{1} \mathrm{KNO}_{3}+s_{2} \mathrm{C}+s_{3} \mathrm{~S} \rightarrow \mathrm{s}_{4} \mathrm{~K}_{2} \mathrm{SO}_{4}+s_{5} \mathrm{~K}_{2} \mathrm{CO}_{3}+s_{6} \mathrm{CO}_{2}+s_{7} \mathrm{CO}+s_{8} \mathrm{~N}_{2} ;(14) \\
s_{1} \mathrm{KMnO}_{4}+s_{2} \mathrm{H}_{2} \mathrm{~S} \rightarrow \mathrm{s}_{3} \mathrm{~K}_{2} \mathrm{SO}_{4}+s_{4} \mathrm{~K}_{2} \mathrm{~S}_{2} \mathrm{O}_{3}+s_{5} \mathrm{~S}+s_{6} \mathrm{MnS}+s_{7} \mathrm{H}_{2} \mathrm{O} ;(15) \\
s_{1}\left(\mathrm{Cr}\left(\mathrm{H}_{2} \mathrm{NCONH}_{2}\right)_{6}\right)_{4}\left(\mathrm{Cr}(\mathrm{CN})_{6}\right)_{3}+s_{2} \mathrm{KMnO}_{4}+s_{3} \mathrm{HCl} \rightarrow s_{4} \mathrm{~K}_{2} \mathrm{Cr}_{2} \mathrm{O}_{7} \\
+s_{5} \mathrm{CO}_{2}+s_{6} \mathrm{KNO}_{3}+\mathrm{s}_{7} \mathrm{MnCl}_{2}+s_{8} \mathrm{KCl}+s_{9} \mathrm{H}_{2} \mathrm{O} .
\end{gathered}
$$

It is easy to see that in chemical the equation (13) the difference between the numbers of unique structural units $\{K, N, O, C\}$ and the desired stoichiometric coefficients is $6=\mid \underbrace{\operatorname{card}\{K, N, O, C\}}_{4}-\underbrace{\operatorname{card}\left(\left\{s_{j}\right\}_{j=1,10}\right.}_{10}) \mid$; in the equation (14) this 
difference is $3=|\underbrace{\operatorname{card}\{K, N, O, C, S\}}_{5}-\underbrace{\operatorname{card}\left(\left\{s_{j}\right\}_{j=1,8}\right)}_{8}|$; in the equation (15) $2=\mid \underbrace{\operatorname{card}\{K, M n, O, H, S\}}_{5}-\underbrace{\operatorname{card}\left(\left\{s_{j}\right\}_{j=1,7}\right.}_{7}) ;$ and in the equation (16), as in the equations (7) and (12) from Examples 1 and 2, respectively, this difference is $1=|\underbrace{\operatorname{card}\{C r, H, N, C, O, K, M n, C l\}}_{8}-\underbrace{\operatorname{card}\left(\left\{s_{j}\right\}_{j=1,9}\right)}_{9}|$. Recall that the number of unique structural units is the number of equations in the constructed system with respect to the desired stoichiometric coefficients, and, therefore, the number $\mid \operatorname{card}(\{$ set of base units $\})-\operatorname{card}\left(\left\{s_{j}\right\}_{j \in \mathbb{N}}\right) \mid$ determines whether the constructed system with respect to the unknown stoichiometric coefficients is a normal, underdetermined or overdetermined system (obviously, provided that the equations of the system are linearly independent).

The system of linear algebraic equations for the desired stoichiometric coefficients, constructed on the basis of the material balance law, is

- an underdetermined system for the reaction (13):

$$
\underbrace{\left(\begin{array}{cccccccccc}
1 & 0 & -2 & -2 & -1 & 0 & 0 & 0 & 0 & 0 \\
1 & 0 & 0 & 0 & -1 & 0 & 0 & -1 & -2 & 0 \\
3 & 0 & -4 & -3 & -2 & -3 & -2 & -1 & 0 & -2 \\
0 & 1 & -1 & -1 & 0 & -1 & -1 & 0 & 0 & 0
\end{array}\right)}_{A_{4 \times 10}} Z_{10 \times 1}=\underbrace{0}_{U_{4 \times 1}}, Z_{10 \times 1}=\left\{s_{j}\right\}_{j=1,10} ;
$$

- an underdetermined system for the reaction (14):

$$
\underbrace{\left(\begin{array}{cccccccc}
1 & 0 & 0 & -2 & -2 & 0 & 0 & 0 \\
1 & 0 & 0 & 0 & 0 & 0 & 0 & -2 \\
3 & 0 & 0 & -4 & -3 & -2 & -1 & 0 \\
0 & 1 & 0 & 0 & -1 & -1 & -1 & 0 \\
0 & 0 & 1 & -1 & 0 & 0 & 0 & 0
\end{array}\right)}_{A_{5 \times 8}} Z_{8 \times 1}=\underset{U_{5 \times 1}^{*}}{0}, Z_{8 \times 1}=\left\{s_{j}\right\}_{j=1,8}
$$

- an underdetermined system for the reaction (15): 
Aleksejeva \& Guseynov, 2021. To the Issue of Finding the Stoichiometric Coefficients in the Chemical Reactions

$$
\underbrace{\left(\begin{array}{ccccccc}
1 & 0 & -2 & -2 & 0 & 0 & 0 \\
1 & 0 & 0 & 0 & 0 & -1 & 0 \\
4 & 0 & -4 & -3 & 0 & 0 & -1 \\
0 & 1 & 0 & 0 & 0 & 0 & -1 \\
4 & 0 & -4 & -3 & 0 & 0 & -1
\end{array}\right)}_{A_{5 \times 7}} Z_{7 \times 1}=\underbrace{0}_{U_{5 \times 1}}, Z_{7 \times 1}=\left\{s_{j}\right\}_{j=1, \overline{7}} ;
$$

- a normal system for reaction (16) (because we can add to it equation $s_{1}=p$, where $p$ is any positive number, for example, 1$)$ :

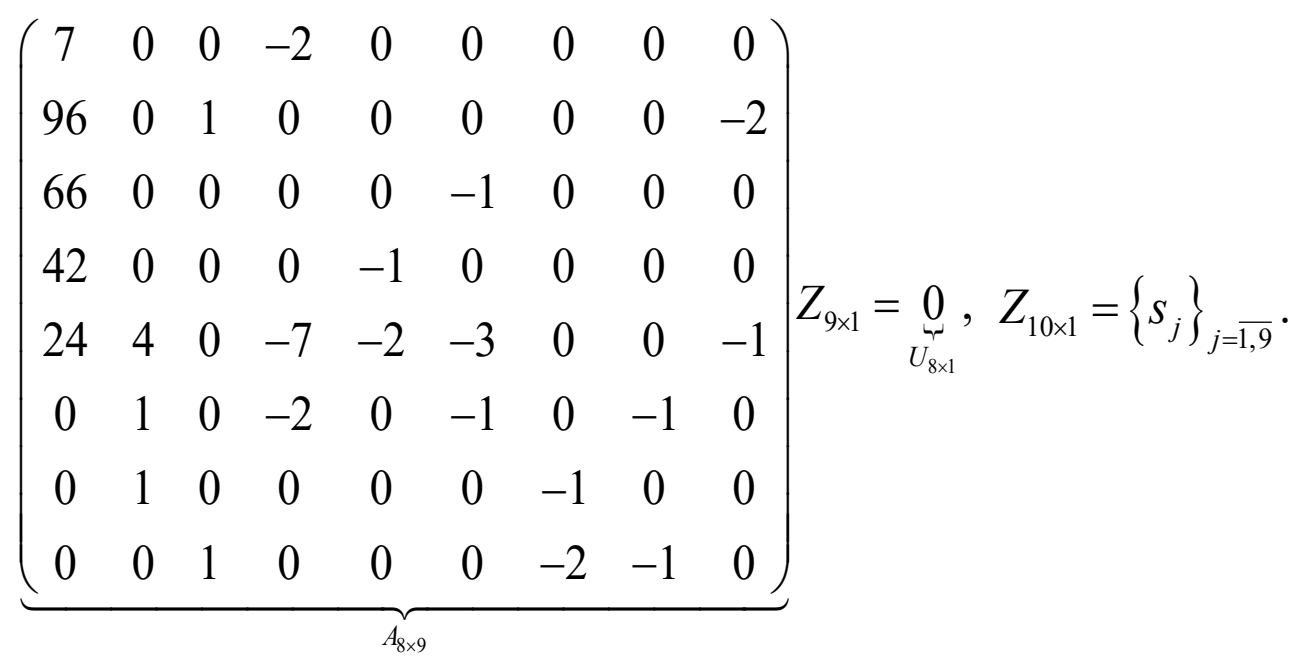

System (20), after supplementing it with equation $s_{1}=p$, where as $p$ one can take any positive number (for example, $p=1$ ), can be solved manually or by some direct analytical or numerical method, for example, Gaussian elimination method, or Cramer's rule, or inverse matrix solution. As a result, we get $s_{1}=10$, $s_{2}=1176, s_{3}=2798, s_{4}=35, s_{5}=420, s_{6}=660, s_{7}=1176, s_{8}=446, s_{9}=1879$. Therefore, the balanced reaction equation (15) has the form

$$
\begin{aligned}
& 10\left(\mathrm{Cr}_{(}\left(\mathrm{H}_{2} \mathrm{NCONH}_{2}\right)_{6}\right)_{4}\left(\mathrm{Cr}(\mathrm{CN})_{6}\right)_{3}+1176 \mathrm{KMnO}_{4}+2798 \mathrm{HCl} \rightarrow 35 \mathrm{~K}_{2} \mathrm{Cr}_{2} \mathrm{O}_{7} \\
& +420 \mathrm{CO}_{2}+660 \mathrm{KNO}_{3}+1176 \mathrm{MnCl}_{2}+446 \mathrm{KCl}+1879 \mathrm{H}_{2} \mathrm{O} .
\end{aligned}
$$

Since systems (17)-(19) are underdetermined systems, they cannot be solved by the usual direct analytical or numerical methods. In the article (Kulibaba, 2010) (we would like to note that this article was written, unfortunately, somewhat carelessly and there are many mathematical typos), the underdetermined systems (18) and (19) were not solved, or rather, there is an unsuccessful attempt to solve their logical and exhaustive way, however, as a result of this attempt, not entirely correct results were obtained. The implementation of the regularizing algorithm proposed in the previous section in Mathcad, version 14.0.0.163, allows us to determine the correct values of the desired stoichiometric coefficients of chemical 
equations (14) and (15) (when performing Step 1 of the proposed algorithm, we perturbed the elements of the main matrices and the right-hand sides of systems (18) and (19) using the built-in function $\operatorname{rnd}(x)$, where we put $x=0.2)$. Namely:

- a normal stable pseudosolution of the system (18) is vector (i.e., a solution to the system (4), see Fig. 2)

$z_{-} \alpha(\alpha)=$\begin{tabular}{|c|c|}
\hline & 1 \\
\hline 1 & 4.00328 \\
\hline 2 & 3.99871 \\
\hline 3 & 0.99969 \\
\hline 4 & 0.99877 \\
\hline 5 & 1.00016 \\
\hline 6 & 1.99999 \\
\hline 7 & 1.00111 \\
\hline 8 & 1.99899 \\
\hline
\end{tabular}

whose coordinates, as has been said many times earlier, should be rounded to the nearest natural numbers: as a result, we get that $s_{1}=4, s_{2}=4, s_{3}=1, s_{4}=1, s_{5}=1$, $s_{6}=2, s_{7}=1, s_{8}=2$, and, therefore, the correctly balanced reaction equation (14) is the equation

$$
4 \mathrm{KNO}_{3}+4 \mathrm{C}+\mathrm{S} \rightarrow \mathrm{K}_{2} \mathrm{SO}_{4}+\mathrm{K}_{2} \mathrm{CO}_{3}+2 \mathrm{CO}_{2}+\mathrm{CO}+2 \mathrm{~N}_{2}
$$
which in article (Kulibaba, 2010) looked like

$$
12 \mathrm{KNO}_{3}+8 \mathrm{C}+5 \mathrm{~S} \rightarrow 5 \mathrm{~K}_{2} \mathrm{SO}_{4}+\mathrm{K}_{2} \mathrm{CO}_{3}+6 \mathrm{CO}_{2}+\mathrm{CO}+6 \mathrm{~N}_{2},
$$

in which the law of material balance is formally fulfilled.

- a normal stable pseudosolution of system (19) is a vector (i.e., a solution to system (4), see Fig. 2)

$$
z_{-} \alpha(\alpha)=\begin{array}{|r|r|}
\hline & \multicolumn{1}{|c|}{1} \\
\hline 1 & 3.99663 \\
\hline 2 & 8.98872 \\
\hline 3 & 0.99999 \\
\hline 4 & 1.0003 \\
\hline 5 & 1.99997 \\
\hline 6 & 3.99999 \\
\hline 7 & 9.00142 \\
\hline
\end{array}
$$

which allows you to write a correctly balanced equation of the chemical reaction (15)

$$
4 \mathrm{KMnO}_{4}+9 \mathrm{H}_{2} \mathrm{~S} \rightarrow \mathrm{K}_{2} \mathrm{SO}_{4}+\mathrm{K}_{2} \mathrm{~S}_{2} \mathrm{O}_{3}+2 \mathrm{~S}+4 \mathrm{MnS}+9 \mathrm{H}_{2} \mathrm{O}
$$

instead of a not entirely correct equation (at least, it is not an equation with, as a rule, the required minimum stoichiometric coefficients)

$$
10 \mathrm{KMnO}_{4}+22 \mathrm{H}_{2} \mathrm{~S} \rightarrow 3 \mathrm{~K}_{2} \mathrm{SO}_{4}+2 \mathrm{~K}_{2} \mathrm{~S}_{2} \mathrm{O}_{3}+5 \mathrm{~S}+10 \mathrm{MnS}+22 \mathrm{H}_{2} \mathrm{O}
$$

from article (Kulibaba, 2010).

In (Kulibaba, 2010), system (17) is not solved, and it is argued that (we quote) "... The system has many solutions, but it is impossible to find a 
mathematical way to solve it. The only option left is to orally select the coefficients ...".

Application of the proposed regularizing algorithm (4)-(6) to system (17) gives us the following normal pseudo-solution (the algorithm is implemented in Mathcad, version 14.0.0.163, while the elements of the main matrix and the righthand side of system (17) were perturbed using the built-in function where we put again $x=0.2$ ):

$$
z_{-} \alpha(\alpha)=\begin{array}{|c|c|}
\hline & 1 \\
\hline 1 & 9.73782 \\
\hline 2 & 6.64992 \\
\hline 3 & 1.88514 \\
\hline 4 & 1.92046 \\
\hline 5 & 1.77621 \\
\hline 6 & 1.86405 \\
\hline 7 & 1.62960 \\
\hline 8 & 2.77704 \\
\hline 9 & 0.85882 \\
\hline 10 & 0.87763 \\
\hline
\end{array}
$$

Before carrying out the necessary rounding off of the coordinates of the obtained pseudo-solution vector (21) to the nearest natural numbers, let us pay attention to the "dangerous" coordinates, which are the second and seventh coordinates. Why are these coordinates "dangerous"? The fact is that if instead of the values 6.64992 and 1.6296 of these coordinates there were values, for example, 6.5 and 1.5, respectively, then the necessary rounding of them to the nearest natural numbers would give us four alternatives: 6 and 1, or 6 and 2, or 7 and 1 , or 7 and 2, and, therefore, all these four alternatives must be taken into account in turn in equation (13), as a result of which there is a single correct set of values of the sought stoichiometric coefficients:

$$
10 \mathrm{KNO}_{3}+7 \mathrm{~S} \rightarrow 2 \mathrm{~K}_{2} \mathrm{SO}_{4}+2 \mathrm{KSO}_{3}+2 \mathrm{KNO}_{2}+2 \mathrm{SO}_{3}+2 \mathrm{NO}+3 \mathrm{~N}_{2}+\mathrm{O}_{2}+\mathrm{SO}_{2} .
$$

In the general case, if the dimension of the found pseudosolution vector is $n \times 1$ (i.e. if the number of unknown stoichiometric coefficients is $n$ ) and, if all coordinates of this pseudo-solution are "dangerous" in the above sense (one cannot exclude the occurrence of such cases!), then there are $2^{n}$ alternatives, which greatly complicates the determination of the unique correct set of values of the unknown stoichiometric coefficients in the equation of the considered chemical reaction. It seems to us that the solution to the arisen brute force problem of $2^{n}$ alternatives can be significantly facilitated (in the sense of simplifying calculations) if we use the apparatus of rich theory of Boolean functions, in particular, algorithms for finding the minimal disjunctive form of both a completely defined Boolean function and an incompletely defined Boolean function. However, this paper will not investigate the possibility of using the apparatus of the theory of Boolean functions for computational simplification 
when solving the above-described enumeration problem for $2^{n}$ alternatives of rounding the coordinates of the obtained pseudo-solution vector. In conclusion, we just add that in any case, even brute forcing through $2^{n}$ alternatives is extremely easier, than brute forcing through $\prod_{j=1}^{n} \bar{m}_{j}$ alternatives of values of stoichiometric coefficients or even a chemical-logical search among $\prod_{j=1}^{n}\left(\bar{m}_{j}-\underline{m}_{j}+1\right)$ alternatives of values of stoichiometric coefficients, where natural numbers $\underline{m}_{j}$ and $\bar{m}_{j}$ denote estimated lower and upper boundaries of the stoichiometric coefficient $s_{j}, j \in\{1, \ldots, n\}$. For example, let $n=5$, and let it be established from a chemical-logical way that the stoichiometric coefficient $s_{1}$ can take one of the values from the set $\{3, \ldots, 10\}$ of possible values, $s_{2}$ - from $\{1, \ldots, 5\}, s_{3}-$ from $\{6, \ldots, 11\}, s_{4}-$ from $\{4, \ldots, 7\}, s_{5}-$ from $\{3, \ldots, 5\}$; then we get 2880 alternatives in chemical-logical enumeration, which is 90 times more than $2^{5}$ alternatives, which arise due to the above-described ambiguity of rounding the coordinates of the found pseudo-solution.

Remark 4. If we at least visually compare the pseudosolution (21) with the found pseudosolutions from the previous examples, then it is easy to see that (21) deviates more from the true solution, which we already know (see the coefficients in equation (22)). This is indeed the case, as evidenced by the relatively large absolute (order $10^{-1}$ ) and relative (order $10^{-2}$ ) of pseudo-solution error (21), namely, $\|Z-\underbrace{Z_{\text {Reg. }}^{\alpha} \|}_{z_{-} \alpha(\alpha)}\| 0.70795, \frac{\left\|Z-Z_{\text {Reg. } \|}^{\alpha}\right\|}{\left\|Z_{\text {Reg. }}^{\alpha}\right\|} \approx 0.05515$. It is intuitively clear that $\left\|Z-Z_{\text {Reg. }}^{\alpha}\right\| \sim \mid \operatorname{card}(\{$ set of base units $\})-\operatorname{card}\left(\left\{s_{j}\right\}_{j \in \mathbb{N}}\right) \mid$, where the symbol $\sim$ means proportionality, e. the greater the difference between the numbers of the desired stoichiometric coefficients and equations in the system of algebraic equations (3), the lower the resolution becomes (in other words, the efficiency of application) of the proposed regularizing algorithm, and, thereby, the values of the absolute and relative errors of the found pseudosolution of the system (3): one of the authors of this work, while still a 4th year student of Lomonosov Moscow State University, investigated the essence of this proportionality when solving operator equations of the first kind, which include, in particular, the system of linear algebraic equations (see Dmitriev \& Guseynov, 1995). EOR 
Aleksejeva \& Guseynov, 2021. To the Issue of Finding the Stoichiometric Coefficients in the Chemical Reactions

\section{Discussion}

Obviously, the problem of determining stoichiometric coefficients in equations of chemical reactions arises not only in the corresponding academic disciplines of chemistry taught in secondary schools and higher educational institutions - at least, it is an imprescriptible "routine" task in chemical industries, some of the main questions of which are: - how much raw material should be taken to maintain a given productivity taking into account the degree of conversion of reagents, process selectivity and losses? - how much energy is needed for the efficient implementation of simple and complex chemical processes? The authors of this work, academic personnel, have never been associated with chemical industries and technologies, and we do not know exactly how automated material and energy calculations in chemical industries and technologies in various countries are (at least in the developed countries of the European Union and North America). Even if we assume that there is full automation, then the mathematical method proposed in this work, which, without much difficulty, can be automated (not using Mathcad software, as it was done in this work due to its intuitive and easy-to-use interface (see Remark 2), but through software development using an Integrated development environment), can be considered as an alternative full-fledged innovative approach that does not require deep knowledge of chemistry (at least knowledge of oxidation numbers theory), especially in relation to redox reactions, in the equations of which three methods are mainly used to find the stoichiometric coefficients - the electron-balance method, the ion-electron method (also called the half-reaction method), the method of Arcesio Garcia (see Garcia, 1987; note that there is still no proper scientific justification for this method), the use of which requires memorizing some chemical facts and operating them, for example, memorizing the oxidation states of some elements, the behavior of oxidants and reducing agents in different environments, etc. Nevertheless, in Remark 1 we emphasized that this work is intended primarily for teachers of a traditional chemistry course and for high school students taking such a course. Natural questions arise: - whether the students of senior grades of general education schools are able to assimilate (independently or under the guidance of teachers) the content of this work; whether, generally speaking, students of general education schools need to know which types of systems of linear algebraic equations exist? - whether they need to study 2-3 direct methods for solving normal systems (at least the Gaussian elimination method and/or the Cramer's rule, and/or the inverse matrix solution)? - whether they need to understand what problems may arise when implementing these methods on computers (i.e., when they are numerically implemented)? - whether they need to be able to apply the gained knowledge to 
solve practical/real problems, for example, to solve the problem of determining stoichiometric coefficients in equations of chemical reactions.

Our answers to all these questions are unequivocally affirmative. At one time, studying under a 10-year educational system, in the 7th grade within the framework of the "Algebra" subject, authors studied the above-mentioned direct methods for solving normal systems of linear algebraic equations (and manually solved systems with 10 equations and unknowns); in the 7th grade, within the framework of the "Algebra" subject, they studied types and properties of matrices and vectors/arrays and operations on them; in the 8th grade, within the framework of the same subject, they studied underdetermined and overdetermined systems of linear equations and were able to find bases, basic solutions and basic admissible solutions of such systems; the second author, in the 8-10th grades, at extra optional lessons, studied the elements of mathematical modeling, within which the studied problems were reduced to problems of game theory, linear programming and graph theory; the second author, in the 10th grade, within the framework of an extra optional lesson, studied elements of the theory of inverse problems - a class of problems in which, according to known consequences, it is required to determine the causes; etc. It can be added that in grades 9-10 in the framework of the subject "Mathematics" we studied complex numbers, limits, differential and integral calculus, elements of analytical geometry, elements of the theory of probability and mathematical statistics, etc. We emphasize once again that all this was carried out in general education schools with a 10-year educational system. Why is it impossible to study at least the elements of linear algebra at a good level in general education schools with a 12-year educational system?! Of course, in almost every general education school there are several schoolchildren who, for one reason or another (mainly under the influence of relatives and friends), will try to deepen their knowledge, but this is not about units, but about the massiveness of obtaining deep knowledge. Maybe deep education is not needed? - if so, who exactly does not need it and for what reasons? After all, it is almost an axiom that the main component of sustainable and dynamic development of any state is development and realization of the intellectual potential of the nation!

Let us return to the approach proposed in this work for determining stoichiometric coefficients in equations of chemical reactions of any type. Let's give a simple typical example, considering a simple redox reaction with reagents - oxidizing agent $\mathrm{KMnO}_{4}$, environment $\mathrm{H}_{2} \mathrm{O}$, reducing agent $\mathrm{Na}_{2} \mathrm{SO}_{3}$, and products $\mathrm{MnO}_{2}, \mathrm{KOH}, \mathrm{Na}_{2} \mathrm{SO}_{4}$, that is, let us consider the chemical equation

$$
\mathrm{s}_{1} \mathrm{KMnO}_{4}+\mathrm{s}_{2} \mathrm{H}_{2} \mathrm{O}+\mathrm{s}_{3} \mathrm{Na}_{2} \mathrm{SO}_{3} \rightarrow \mathrm{s}_{4} \mathrm{MnO}_{2}+s_{5} \mathrm{KOH}+s_{6} \mathrm{Na}_{2} \mathrm{SO}_{4} \text {, }
$$

where $\left\{s_{i}\right\}_{i=1, \overline{1}}$ are the desired stoichiometric coefficients. 
Application of the half-reaction method to the considered chemical reaction generates the following stages (this method is based on preparation of ionic equations for oxidation and reduction processes with their subsequent summation into a general equation):

- constructing the ion-molecular equation of each half-reaction, taking into account the transformation of permanganate ion in a neutral medium into manganese dioxide:

$$
\begin{gathered}
\mathrm{MnO}_{4}^{-}+\mathrm{H}_{2} \mathrm{O} \rightarrow \mathrm{MnO}_{2}+\mathrm{OH}^{-}, \\
\mathrm{SO}_{3}^{2-}+\mathrm{H}_{2} \mathrm{O} \rightarrow \mathrm{SO}_{4}^{2-}+\mathrm{H}^{+}
\end{gathered}
$$

- obtaining electron-ion equations by arranging the coefficients in each halfreaction and determining number of electrons participating in it:

$$
\left.\begin{array}{c}
\mathrm{MnO}_{4}^{-}+2 \mathrm{H}_{2} \mathrm{O}+3 \bar{e} \rightarrow \mathrm{MnO}_{2}+4 \mathrm{OH}^{-} \mid \text {electrons: } 3 \text {; coefficient: } 2, \\
\mathrm{SO}_{3}^{2-}+\mathrm{H}_{2} \mathrm{O}-2 \bar{e} \rightarrow \mathrm{SO}_{4}^{2-}+2 \mathrm{H}^{+} \mid \text {electrons: } 2 \text {; coefficient: } 3 ;
\end{array}\right\} \Rightarrow
$$

- obtaining ion-molecular equation of the redox reaction by summing electron-ion equations obtained in the previous stage and reducing similar terms:

$$
2 \mathrm{MnO}_{4}^{-}+\mathrm{H}_{2} \mathrm{O}+3 \mathrm{SO}_{3}^{2-} \rightarrow 2 \mathrm{MnO}_{2}+2 \mathrm{OH}^{-}+3 \mathrm{SO}_{4}^{2-}
$$

- constructing the desired molecular equation according to the obtained ionmolecular equation:

$$
2 \mathrm{KMnO}_{4}+\mathrm{H}_{2} \mathrm{O}+3 \mathrm{Na}_{2} \mathrm{SO}_{3} \rightarrow 2 \mathrm{MnO}_{2}+2 \mathrm{KOH}+3 \mathrm{Na}_{2} \mathrm{SO}_{4} .
$$

Now we apply a mathematical approach to equation (23) of the considered redox reaction. Since in equation (23) number of unique structural units is 6 $(K, M n, O, H, N a, S)$ and number of required stoichiometric coefficients is also 6, we obtain a square system of linear algebraic equations

$$
\left\{\begin{array}{l}
s_{1}=s_{5}, \\
s_{1}=s_{4}, \\
4 \cdot s_{1}+s_{2}+3 \cdot s_{3}=2 \cdot s_{4}+s_{5}+4 \cdot s_{6}, \\
2 \cdot s_{2}=s_{5}, \\
2 \cdot s_{3}=2 \cdot s_{6}, \\
s_{3}=s_{6},
\end{array}\right.
$$

in which the last two equations coincide and, therefore, one of them should be excluded from consideration. It is quite easy to express all the coefficients $\left\{s_{i}\right\}_{i=\overline{2,6}}$ 
through $s_{1}: s_{2}=\frac{1}{2} \cdot s_{1}, s_{3}=\frac{3}{2} \cdot s_{1}, s_{4}=s_{1}, s_{5}=s_{1}, s_{6}=\frac{3}{2} \cdot s_{1}$. Since stoichiometric coefficients $\left\{s_{i}\right\}_{i=1,6}$ must be natural numbers, the minimum value $s_{1}$ guaranteeing naturalness of $\left\{s_{i}\right\}_{i=2,6}$, is 2 , that is, we put $s_{1}=2$. Consequently, $s_{2}=1, s_{3}=3$, $s_{4}=2, s_{5}=2, s_{6}=3$. Taking these values into account in equation (23) gives us equation (24), which was established by a chemical method - the half-reaction method.

In the context of the simple example just considered, two questions immediately arise: (a) do authors of this work claim that the proposed mathematical method is better than the chemical method (the word "best" can mean "simple" and/or "easy" and/or "visual", etc.)? (b) what about the "cumbersome" algorithm proposed in this work? - after all, the applied mathematical method (compilation and solution of a system of simple linear equations (25)) is not the "cumbersome" algorithm that is proposed. Our answers to these questions are as follows: (a) no, we do not claim this - our work did not carry out a comparative analysis of the mathematical and chemical methods from the point of view of "better or worse", the proposed mathematical approach can be perceived as a full-fledged alternative method that can be easily implemented on any of the modern softwares, in particular, in Mathcad software with a convenient and intuitive interface (see Remark 2), and we are sure that students of secondary schools (in particular, in Latvia) are quite capable of fully mastering the proposed algorithm; (b) in the considered simple example, the number of unique chemical structural units coincides with the number of the desired stoichiometric coefficients, and, therefore, the resulting system of equations (generally speaking, the obtained system is not always as simple as system (25)) could be solved manually or by one from direct methods on a computer; if the resulting system of equations is underdetermined (the number of unique structural units is greater than the number of desired stoichiometric coefficients) or overdetermined (the number of unique structural units is less than the number of desired stoichiometric coefficients), and the difference between the numbers of equations and unknowns is more than 2, then it is impossible to solve them by conventional direct analytical or numerical methods, and then the algorithm proposed in this work acts as an "irreplaceable key" for finding stoichiometric coefficients in the equations of chemical reactions, which can be arbitrarily complex (especially in modern chemical industries).

In conclusion of this section, we would like to note that authors tried to reduce the large amount of this work, however, unfortunately, we could not do this without prejudice to understanding and complete assimilation of the proposed algorithm (except for this section, which can be removed). However, the authors 
do not exclude that this reason for not reducing the amount of this work is subjective.

\section{Conclusions}

In this paper, to determine the stoichiometric coefficients in the equations of chemical reactions, we propose a regularizing algorithm (consisting of two steps), which is based on the fundamental concept of Tikhonov regularization. The proposed algorithm finds a normal stable pseudosolution of a system of linear algebraic equations constructed by applying the material balance law. The examples implemented in Mathcad software, version 14.0.0.163, demonstrate the applicability of this algorithm to both normal systems and underdetermined or overdetermined systems. Further, this paper describes a mechanism for reducing the problem of determining stoichiometric coefficients in the equations of chemical reactions to systems of linear algebraic equations. Using examples of specific chemical reactions, including redox reactions, both the aforementioned mechanism for constructing a system of linear algebraic equations and ensuring the implementation of both steps of the proposed regularizing algorithm are shown. Finally, this paper discusses, in a way, the limits of the possibility of applying the proposed regularizing algorithm to the problem of mathematical determination of stoichiometric algorithm) to the nearest natural numbers, there is an ambiguity in the choice of the direction of rounding (what to choose: floor function or ceiling function?): such situations arise when the difference between the numbers of unknowns (stoichiometric coefficients) and system equations is significant.

\section{Acknowledgements}

The authors of this work would like to express their gratitude to the 4th year student of the Faculty of Mechanics and Mathematics of Lomonosov Moscow State University Ruslans Aleksejevs and Ms.Sc. Comp. Sergey A. Andreev for valuable scientific advices concerning implementation of the proposed regularizing algorithm in softwares, especially in Mathcad.Also, the authors of this work express their gratitude to the anonymous reviewers for their critical comments and valuable advice, which we took into consideration: among other things, a "Discussion" section was added to the article, which was missing before the review.

This work was carried out within the partial financial support from the European Regional Development Fund Project No. 1.1.1.5/018 "Promotion of research, innovation and international cooperation in science at Liepaja University". 


\section{References}

Abkin, G.L. (1971). Methodology for solving problems in chemistry. A guide for teachers. Moscow: "Education" Publishing House.

Adamishvili, I.P. \& Gambashidze, N.N. (1984). Algebraic way of arranging coefficients in the equations of reactions. Chemistry at School, 4, 33-36.

Andreyev, S.A. \& Guseynov, Sh.E. (2013). Regularizing algorithms for diagnosing: Applied to gas turbine engines in operation. Saarbrucken: LAP Publishing.

Anton, H. \& Rorres, Ch. (2014). Linear Algebra with Applications. New York: WileyPLUS.

Berg, L.G., Gromakov, S.D., Zoroatskaya, I.V., Averko-Antonovich, I.N. (1959). Methods for the selection of coefficients in chemical equations. Kazan: Kazan State University Press.

Blakley, G.R. (1982). Chemical equation balancing: A general method which is quick, simple, and has unexpected applications. Journal of Chemical Education, 59(9), 728-734.

Dmitriev, V.I. \& Guseynov, Sh.E. (1995). On matching the resolving power and detailedness of inverse problems solution. Moscow State University Herald, Series 15: Computational Mathematics and Cybernetics, 1, 24-27.

Gantmacher, F.R. (1967). Theory of Matrices. Moscow: Nauka Publishing.

Garcia, A. (1987). A new method to balance chemical equations. Journal of Chemical Education, 64(3), 247-248.

Guseynov, Sh. E., Aleksejevs, R., Aleksejeva, J. V. \& Gasimov, Y. S. (2017). Evaluating attractiveness of the central and the eastern European countries by using index approach for the strategic decision making process related to expansion of the financial service markets. Advanced Mathematical Models \& Applications, 2(3), 167-214.

Guseynov, Sh. E., Urbah, A. I. \& Andreyev, S. A. (2015). On one approach for stable estimate of technical system efficiency. Proceedings of the 10th International Scientific and Practical Conference "Environment. Technology. Resources", III, Rezekne, Latvia, 100108.

Il'in, V.A. \& Poznyak, E.G. (1999). Linear algebra. Moscow: FizMatLit, 296 p.

Khrustalev, A.F. (1968). On the arrangement of coefficients in chemical equations. Chemistry at School, 5, 67-69.

Krapivin, S.G. (1929). Notes on the method of chemistry. A manual for teachers of II stage schools and technical training schools. Moscow-Leningrad: State Publishing House.

Kulibaba, M.S. (2010). Learn to equalize the equations of chemical reactions. Journal "Chemistry. Everything for the teacher", Pilot issue, $12 \mathrm{p}$.

Kuznetsova, N.E. \& Adrienko, A.L. (1976). Algorithmic prescriptions for the equations of redox reactions as one of the means of enhancing the cognitive activity of students. Chemistry at School, 1, 73-82.

Marinichev, A.N., Sokolova, E.P. \& Zenkevich, I.G. (2014). Stoichiometry of chemical reactions in MAPLE (matrix approach). Advance in Current Natural Sciences, 3, 96-101.

Maxfield, B. (2009). Essential Mathcad for Engineering, Science, and Math. Oxford: Academic Press.

Michałowska-Kaczmarczyk, A.M., Asuero, A.G. \& Tadeusz Michałowski T. (2015). "Why Not Stoichiometry" versus "Stoichiometry - Why Not?" Part I: General Context. Critical Reviews in Analytical Chemistry, 45(2), 166-188.

Pimentel, G.C., Seaborg, G.T., et al. (1963). Chemistry. An Experimental Science. San Francisco: W.H.Freeman and Company. 
Aleksejeva \& Guseynov, 2021. To the Issue of Finding the Stoichiometric Coefficients in the Chemical Reactions

Polyakova, T.M. (1969). Arrangement of coefficients in the equations of redox reactions. Chemistry at School, 1, 70-73.

Rash, A.M. \& Zurbach E.P. (2004). Mathematics Models in Chemistry - An Innovation for Non-Mathematics and Non-Science Majors. PRIMUS, 14(2), 179-191.

Sen, S. K. Agarwal, H. \& Sen S. (2006). Chemical equation balancing: An integer programming approach. Mathematical and Computer Modelling, 44(7-8), 678-691.

Sommer, K. (1975). Wissensspeicher Chemie: Das Wichtigste bis zum Abitur in Stichworten und Übersichten. Berlin: Volk und Wissen.

Tikhonov, A. N. \& Arsenin, V. Ya. (1977). Solutions of Ill-Posed Problems. New York: V.H. Winston \& Sons Publishing.

Turchen, D.N. (2012). Our approach to determining the coefficients in the redox reactions equations. Chemistry in School, 2, 42-48. 